\title{
Lateral Bias In Lingual Bracing DURing SpeEch
}

\author{
Yadong Liu ${ }^{* 1}$, Megan Keough ${ }^{\dagger 1,2}$, Felicia Tong ${ }^{\ddagger 1}$, Oksana Tkachman ${ }^{\star 1}$, Kate Radford ${ }^{\sharp 1}$ and Bryan Gick ${ }^{\nabla 1,2}$ \\ ${ }^{1}$ Department of Linguistics, University of British Columbia, Vancouver, British Columbia, Canada \\ ${ }^{2}$ Haskins Laboratories, New Haven, Connecticut, USA
}

\section{Introduction}

Human bodies exhibit lateral bias between many laterally symmetrical body parts (e.g, hands, feet, eyes, and ears). This bias is thought to increase the efficiency of behaviour and functionality of a system: e.g., laterally biased eyes produce depth perception rather than double vision [1]. Here we examine lateral biases observed in the tongue during speech, a grossly symmetrical structure at a muscular level [2]. During speech, the tongue is bilaterally braced against the back teeth and, for some sounds, the hard palate [3-6]. This bracing assists in the mechanics of certain tongue movements [7] and in the production of medial speech sounds, but is interrupted for the production of some laterals and occasional low vowels [5]. Some previous evidence suggests the movement away from the braced posture may be produced by lowering one side of the tongue first and that the leading side is consistent within speaker $[5,8]$.

We report observations of lateral bias in 6 English speakers, describe its relationship to other lateral biases of those speakers, and suggest possible implications for origins of this bias. If the larger population shows no overall bias (i.e., neither side is represented more than the other), this bias could emerge out of relatively stochastic developmental processes, such as dentition and behavioural optimization during feeding [9]. In such cases, a lateral preference would emerge depending on the environment in which bracing develops. If, however, there appears to be a population-level bias (most speakers prefer one side over the other), this process would instead develop with cortical modulation in much the same way that handedness is thought to arise [10].

\section{Methods}

\subsection{Materials and Procedures}

Video footage was recorded for 6 native English speakers using a video camera (Sony Cyber-shot DSC-RX100) held by an instrument arm attached to the chair where the participant was seated. Each participant read aloud a oneminute-long English passage while biting on two $5 \mathrm{~mm}$ bite blocks (stacked narrow wooden tongue depressors). The passage was designed to minimize labial sounds to prevent lip closure from obstructing the camera view. The bite blocks kept the mouth open throughout reading, allowing a clear image of tongue movements. An LED light attached to each bite block illuminated the oral cavity.

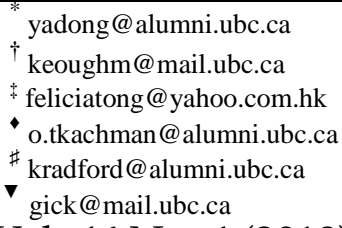

56 - Vol. 46 No. 4 (2018)
Participants were seated with their head stabilized against a headrest with the bite block positioned between the top and bottom molars on each side of the mouth. The passage was read twice. To obtain a baseline resting position for each participant's tongue, the video recording began a few seconds prior to the reading.

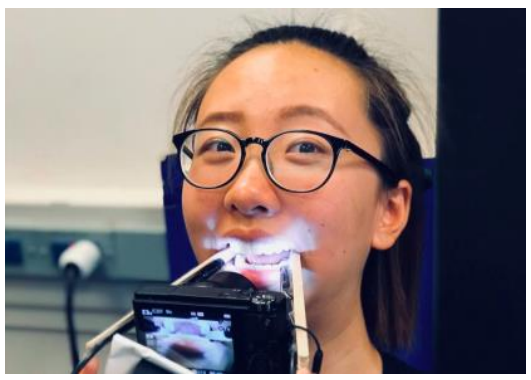

Figure 1: Experimental setup showing participant seated and fitted with bilateral bite block.

\subsection{Analysis}

Each video recording was digitized into kymographic image sequences via ImageJ [11]. Images were stacked and analyzed in orthogonal views, and kymographs were taken of the center, left and right sides of the tongue (See Figure 2). Each kymograph shows the activity through one slice of the video over time. The center intersect line for the kymograph was positioned between the front teeth; the left and right intersect lines were positioned at the back molar.

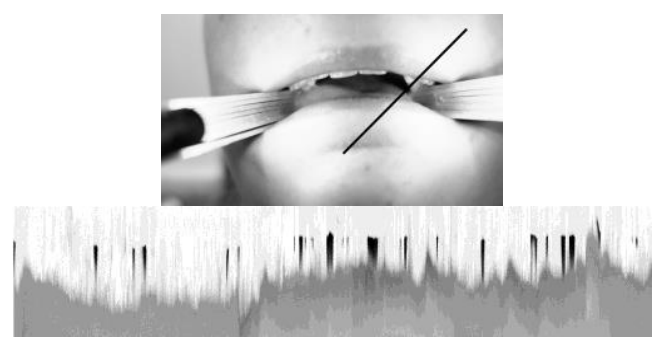

Figure 2. A frame of the video (left), with the black line indicating the location and angle of the slice used produce the kymograph (right). Each column of pixels corresponds to one frame of the video.

Each kymograph was then turned into a black-andwhite image, with white areas corresponding to the tongue and teeth, and black areas corresponding to the open oral cavity, indicating lack of contact between the tongue and teeth. The kymograph was then cropped to contain only active speech. Lastly, a Python script was used to count the number of black pixels in each column of the kymograph (corresponding to the distance of the release from the braced position in one frame of the original video). The movement 
that the sides of the tongue made away from the braced posture has also been referred to as release [5]. The magnitude of release of both sides of the tongue is determined by the area of black regions shown in the kymograph. The black regions indicate no contact between one side of the tongue and the upper teeth, and the vertical magnitude of the black region shows the distance from the side of the tongue and the teeth. Further, the width of the black region presents the duration of the release. Hence, a larger black region illustrates a more significant movement away from the braced posture.

\section{Results}

Figure 3 shows the total amount of release for each side of the tongue for each speaker.

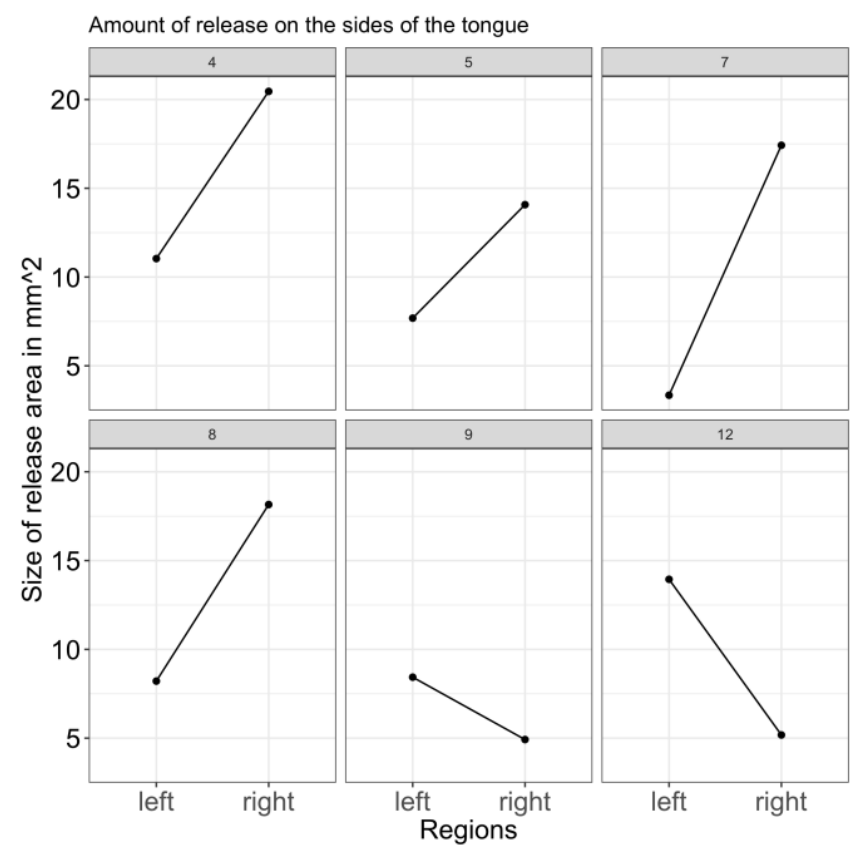

Figure 3: The amount of release of both sides of the tongue for each speaker. Each block shows the result of one participant, Higher values indicate larger black pixel regions on a given side.

The results indicate that one side of the tongue has a larger movement during release than the other side for all participants. Specifically, participants 4, 5, 7, and 8 show greater release for the right side of the tongue, whereas participants 9 and 12 show greater release for the left side. For the two participants with a left side bias, one of them reports being always right-handed while the other reports being left-handed. The rest of participants exhibiting right side biases are all right handed. However, the current sample size is too small to test for a correlation between the lateral bias in the tongue and handedness across participants.

\section{Discussion and conclusions}

Our results indicate that speakers exhibit a lateral bias in the tongue during speech. Participants demonstrated consistent lateral preferences in the tongue when producing lateral liquids and some low vowels. During bite block-braced speech, which exaggerates the movements of the tongue by increasing the vertical distance between the tongue and the palate/teeth, participants showed both a greater number of instances of tongue release as well as a greater magnitude of release for one side of the tongue than another. Given the small number of participants in the current study, it remains unclear whether this bias correlates with handedness. However, the right side is favored for use in 4 out of 6 cases and it is certainly interesting that the directional bias does match for 5 of our 6 speakers. If future research finds that this tendency continues to indicate a population-level bias matching handedness, this will indicate that the emergence of this bias is likely influenced by cortical processes during development. Further research will test whether such a population-level bias exists, so as to determine whether this bias results from similar processes as handedness.

\section{Acknowledgments}

Research funded through NSERC and NIH Grant DC002717 to Haskins Laboratories. We would like to express our thanks to Sophia Luo, who contributed to the methodology of this paper.

\section{References}

[1] McBeath, M. K., \& Sugar, T. G. 2005. Natural selection of asymmetric traits operates at multiple levels. Behav. \& Brain Sci., 28:04.

[2] Sanders, I., \& Mu, L. 2013. A three-dimensional atlas of human tongue muscles. Anat. Record, 296:7, 1102-1114.

[3] Gibbon, F. E., Lee, A., \& Yuen, I. 2010. Tongue-palate contact during selected vowels in normal speech. Cleft Pal.-Craniofac. J., 47:4, 405-412.

[4] Lee, A., Gibbon, F. E., \& Oebels, J. 2015. Lateral bracing of the tongue during the onset phase of alveolar stops: An EPG study. Clin. Ling. \& Phon., 29:3, 236-245.

[5] Gick, B., Allen, B., Roewer-Després, F., \& Stavness, I. 2017. Speaking tongues are actively braced. JSLHR, 60:3, 494.

[6] Narayanan, S.S., Alwan, A.A. and Haker, K. 1997. Toward articulatory-acoustic models for liquid approximants based on MRI and EPG data. Part I. The laterals. JASA, 101:2, pp.1064-1077.

[7] Stone, M. 1991. Toward a model of 3-dimensional tongue movement. J. Phon., 19(3-4), pp. 309-320.

[8] Chen, L., Schellenberg, M., \& Gick, B. 2017. Cross-linguistic bracing: A lingual ultrasound study of six languages. Can. Acoust., 45(3), 186-187.

[9] Hiiemae, K. M., \& Palmer, J. B. 2003. Tongue movements in feeding and speech. Crit. Rev. Oral Biol. \& Med., 14(6), 413-429.

[10] Strauss, E., \& Wada, J. 1983. Lateral preferences and cerebral speech dominance. Cortex, 19(2), 165-177.

[11] Schneider, C. A.; Rasband, W. S. \& Eliceiri, K. W. 2012. NIH Image to ImageJ: 25 years of image analysis. Nature Meth. 9(7): 671-675, PMID 22930834 (on Google Scholar). 